\title{
Flying and Crawling Modes during Surface-Bound Single Wall Carbon Nanotube Growth
}

\author{
Simone Pisana, ${ }^{\dagger}$ Alain Jungen, ${ }^{\ddagger}$ Can Zhang, ${ }^{\dagger}$ Arthur M. Blackburn, ${ }^{\S}$ Renu Sharma,,$"$ \\ Felipe Cervantes-Sodi, ${ }^{\dagger}$ Christoph Stampfer, ${ }^{\ddagger}$ Caterina Ducati, ${ }^{\perp}$ Andrea C. Ferrari, ${ }^{\dagger}$ \\ Christofer Hierold, ${ }^{\ddagger}$ John Robertson, ${ }^{\dagger}$ and Stephan Hofmann $*, \dagger$
}

\begin{abstract}
Department of Engineering, University of Cambridge, CB3 OFA Cambridge, United Kingdom, Micro and Nanosystems, ETH Zurich, 8092 Zurich, Switzerland, Hitachi Cambridge Laboratory, CB3 OHE Cambridge, United Kingdom, LeRoy Eyring Center for Solid State Science, Arizona State University, 85287-1704 Tempe, Arizona, and Department of Materials Science and Metallurgy, University of Cambridge, CB2 $3 Q Z$ Cambridge, United Kingdom
\end{abstract}

Received: July 5, 2007; In Final Form: August 27, 2007

\begin{abstract}
Growth modes of single wall carbon nanotubes are investigated during chemical vapor deposition within an environmental transmission electron microscope and in furnace reactors at different gas pressure and flow conditions. The nanotube pivoting observed by in situ microscopy can be explained by dynamic catalyst crystallite reshaping for base growth. Microfabricated substrate barriers and gaps allow for the distinction between "crawling" and "flying" nanotubes, referring to either a continuous intimate contact with the substrate dominated by van der Waals forces or a mere substrate anchoring of nanotubes held afloat during growth. Flying nanotubes grow unobstructed and straight to millimeter lengths and are susceptible to external forces. Crawling nanotubes are strongly affected by substrate topography. We relate this to tip and base growth regimes and discuss how the growth modes can be controlled.
\end{abstract}

\section{Introduction}

Bottom-up device integration of nanostructures such as single wall carbon nanotubes (SWNTs) requires their position, directional alignment, and structure to be controlled. These requirements can be addressed to various extents in a scalable fashion by catalytic, surface-bound chemical vapor deposition (CVD). Patterning of the catalyst by standard lithography determines the position of the SWNT nucleation sites. ${ }^{1}$ The dimensions of the catalyst nanoparticles and their dynamic interaction with support layers and feeding gases influence the nanotube morphology. ${ }^{2-7}$ Directional control has been demonstrated through gas flow alignment, ${ }^{8,9}$ applied electric fields, ${ }^{10}$ or support interactions, e.g., SWNT growth along stepped surfaces. ${ }^{11}$ Straight, suspended SWNTs have been grown between pillars or microstructured substrates. ${ }^{12-14}$

It is important to note, however, that the alignment mechanism depends on the growth mode of the SWNTs and their nucleation density. External electrostatic fields influence the alignment of the SWNTs only when overcoming their van der Waals (vdW) attraction to the substrate, i.e., when the nanotubes partially fly during growth. ${ }^{15}$ Alignment along step edges and crystal orientations, on the other hand, appears possible only when the SWNTs remain in intimate contact with the substrate. ${ }^{16}$ In the latter example, a base growth mode would additionally lead to shorter nanotubes than tip growth, since for base growth the bodies of SWNTs are required to slide over the support for

* To whom correspondence should be addressed. E-mail: sh315@cam.ac.uk. Telephone: +44(0)1223 748305. Fax: +44(0)1223 748348.

† Department of Engineering, University of Cambridge.

$\doteqdot$ ETH Zurich.

$\S$ Hitachi Cambridge Laboratory.

" Arizona State University.

${ }^{\perp}$ Department of Materials Science and Metallurgy, University of Cambridge. which the interaction force increases with nanotube length. Finally, all horizontal alignment is suppressed if the SWNT nucleation density is so high as to allow growth only perpendicular to the substrate as a result of crowding. ${ }^{17}$

In this paper, we investigate the intimacy of the SWNTsubstrate contact during catalytic CVD in order to establish how the growth environment influences the microscopic force balance. We use substrates with micro-fabricated barriers and gaps and combine environmental transmission electron microscopy (ETEM) of SWNT growth with post-growth analysis of SWNT alignment from various CVD reactors and operating conditions. Previously, ETEM investigations uncovered the nucleation stages of carbon nanofibres ${ }^{2,7}$ and SWNTs. ${ }^{7}$ Here the in situ growth reveals the motion of growing SWNTs. Our analysis provides evidence for two distinct growth modes: "flying", where anchored SWNTs are generally afloat, and "crawling", where the SWNT body is in continuous intimate contact with the substrate during growth. We relate this distinction to the catalytic base and tip growth regimes, allowing us to explain the growth of millimeter-long, straight SWNTs and the influence of external fields used in prior literature.

\section{Experimental Methods}

In situ TEM growth was carried out in a modified Tecnai F20, equipped with a differential pumping scheme (ETEM), operated at $200 \mathrm{kV} .{ }^{18}$ The microscope permits pressures of up to 10 mbar and video sequences can be recorded at 15 frames $\mathrm{s}^{-1}$. Ni catalyst films $(99.9 \%$ purity) were thermally evaporated (base pressure $10^{-6}$ mbar) onto 2000 mesh Cu TEM grids (Agar Scientific) coated with $\mathrm{SiO}_{2}$ nanopowder (Degussa Aerosil Ox50) and a $30 \mathrm{~nm}$ sputtered $\mathrm{SiO}_{2}$ layer. $\mathrm{Ni}$ islands formed upon raising the temperature in vacuum, and growth was initiated in $\mathrm{C}_{2} \mathrm{H}_{2}$ at $8 \times 10^{-3}$ mbar pressure and $600{ }^{\circ} \mathrm{C}$. 
Three substrate types were used for the other growth experiments: perforated $\mathrm{SiO}_{x}$ membranes (SPI supplies), $\mathrm{SiO}_{2}$ capped chips fabricated by surface micromachining of releasable polycrystalline silicon (poly-Si), and $\mathrm{SiO}_{2}$ capped silicon-oninsulator (SOI) with etched-well structures. The wells in the SOI surface were formed by an anisotropic dry etch through a removable polymer mask to a depth of $60 \mathrm{~nm}$, followed by a wet etch in $\mathrm{HF}$ to a total depth of $80 \mathrm{~nm}$, and finally performing a thermal oxidation process to cap the exposed Si surfaces with $\sim 10 \mathrm{~nm}$ of $\mathrm{SiO}_{2}$. Consequently the sidewalls of these wells have a steep undercut profile, with an overhang of $\sim 20 \mathrm{~nm}$. Fe and Co catalyst films were thermally evaporated and patterned by standard photolithography.

Gas flow alignment was investigated in a 2 in. diameter atmospheric pressure quartz tube furnace. Growth with $0.1 \mathrm{~nm}$ Fe catalysts was carried out by heating the samples in an $\mathrm{Ar}$ atmosphere up to $900{ }^{\circ} \mathrm{C}$, followed by a 10 min pretreatment in $200 \mathrm{sccm} \mathrm{H}_{2}$ and growth in a $100 / 500 \mathrm{sccm} \mathrm{H}_{2} / \mathrm{CH}_{4}$ flow for $15 \mathrm{~min}$. Growth with $0.3 \mathrm{~nm}$ Co catalysts was carried out by heating the samples in air up to $850{ }^{\circ} \mathrm{C}$, followed by a 5 min purge in a $4000 \mathrm{sccm}$ Ar flow. After the purge, the hydrocarbon source was introduced by bubbling a 100/1000 $\mathrm{sccm}$ $\mathrm{H}_{2} / \mathrm{Ar}$ flow through a 5:100 $\mathrm{H}_{2} \mathrm{O}$ to ethanol (by volume) solution held at room temperature for $30 \mathrm{~min}$. In all cases, the growth was terminated by purging the furnace atmosphere with a 4000 sccm Ar flow before cooling to room temperature in Ar.

Growth in a static atmosphere was carried out in a lowpressure quartz tube furnace (LPCVD) with a base pressure of $10^{-5}$ mbar. Bimetallic catalyst films ( $1 \mathrm{~nm} \mathrm{Ni}$ over $8 \mathrm{~nm} \mathrm{Al}$ ) were sputtered onto micromachined poly-Si or etched SOI substrates. The chips were transferred in air and subjected to $\mathrm{H}_{2}$ pretreatment at $200 \mathrm{mbar}$ and $850{ }^{\circ} \mathrm{C}$ for $10 \mathrm{~min}$ followed by the growth of SWNTs under $\mathrm{CH}_{4}$ and $\mathrm{H}_{2}$ (3:1) at 200 mbar for $15 \mathrm{~min}$. Heating and cooling were performed under vacuum, and the chamber was opened only after cooling to below $250{ }^{\circ} \mathrm{C}$.

SEM characterization was carried out in a LEO 1530VP or a Zeiss Ultra 55 FEG-SEMs, ex-situ TEM in a JEOL JEM $4000 \mathrm{EX}$ at $400 \mathrm{kV}$.

\section{Results and Discussion}

Figure 1 shows an ETEM image sequence of a SWNT growing from a Ni catalyst particle supported by amorphous silica nanopowder (see video 1 in the Supporting Information). Previous ETEM experiments in identical conditions showed that SWNTs nucleate by lift-off of a carbon cap, thereby following a base growth mechanism. ${ }^{7}$ Cap stabilization and nanotube growth involve the dynamic reshaping of the catalyst nanocrystal itself. Figure 1 shows that the body of the nanotube appears to bend and pivot while growing. When the nanotube touches the neighboring support, growth appears to stop. On the other hand, video 2 in the Supporting Information shows a case where growth continues after the nanotube's attachment on a nearby support, followed by a straightening of its body across the gap. The origin of the pivoting motion will now be discussed in detail. Electron irradiation effects as systematic cause can be ruled out since ex situ grown SWNTs are routinely observed bridging nearest-neighboring features. ${ }^{12,14}$ The incorporation of defects can induce changes in the growth direction at the catalyst/nanotube interface. ${ }^{19,20}$ However, this effect can be excluded for the straight SWNTs observed here and in general whenever high quality SWNTs are grown. Gas flow alignment has been previously observed under atmospheric pressure growth conditions. ${ }^{13,21}$ For CVD in the ETEM, motion mediated by the

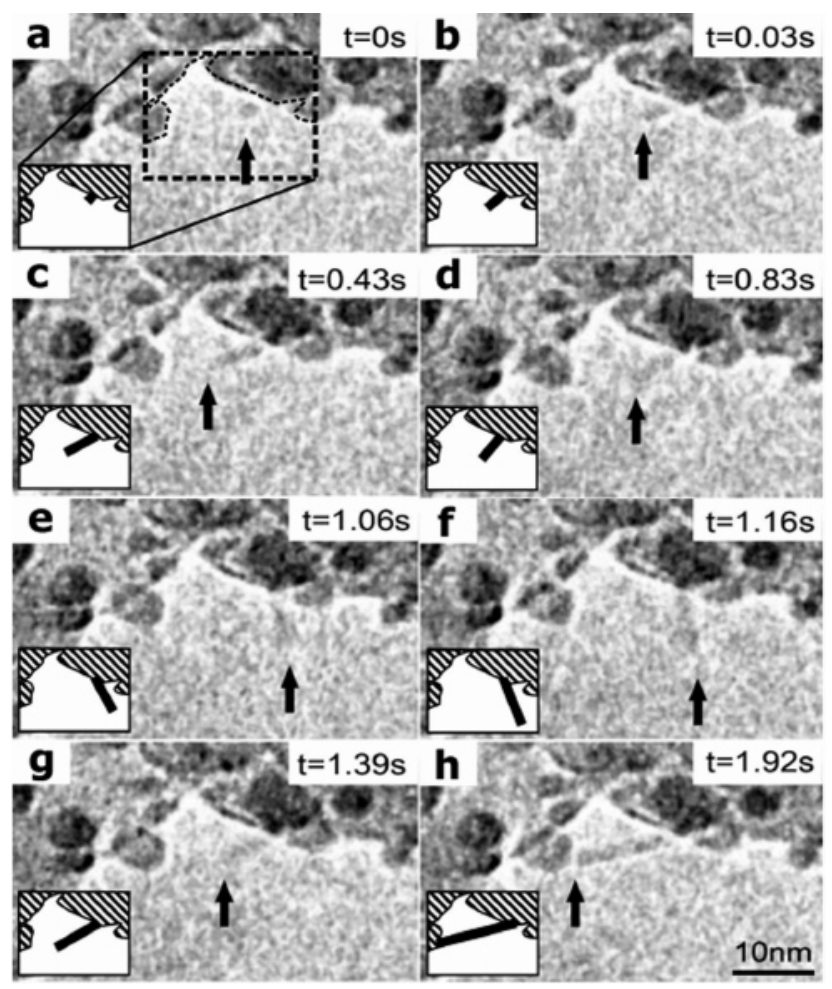

Figure 1. In situ ETEM image sequence of Ni catalyzed SWNT growth (video 1 in the Supporting Information). The light gray area is the $\mathrm{SiO}_{2}$ sputter-coated silica nanopowder; the dark gray areas are the catalyst. The arrows indicate the SWNT tip. Top-right inset: frame time stamp. Bottom-left inset: schematic diagram of the corresponding ETEM image.

gas flow cannot be excluded, though growth takes place at $8 \times$ $10^{-3}$ mbar and the lack of SWNT alignment throughout the substrate indicates that gas mediated alignment is either negligible or the local gas flow direction over the substrate is nonuniform. Temperature-induced motion can be due to the thermal flexing vibrations of the cantilevered nanotube or the rigid pivoting around the nanotube's base. The thermal flexing vibration cannot solely explain the motion in Figure 1, as the calculated average displacement of the cantilever's tip at the growth temperature $(\sim 0.02 \mathrm{~nm})$ is far below the observed value ( $9 \mathrm{~nm}) .^{22}$

Consequently, catalyst particle reshaping appears as the most likely cause of the rigid pivoting at the nanotube's base. Indeed, rapid changes in shape of the crystalline catalyst have been reported in the current growth environment and are consistent with the observed base growth mechanism. ${ }^{7}$

The ETEM data can be related to typical post-growth observations for CVD, such as the bridging across pillars or gaps. Bridging has been reported up to length scales of 100 $\mu \mathrm{m},{ }^{13}$ with its extent varying according to growth conditions. Figure 2a,b shows SWNTs grown in static conditions, i.e., no gas flow, in a LPCVD system at 200 mbar, bridging rounded and sharp microfabricated poly-Si tips. Statistical observations show that the number of bridging SWNTs rapidly decreases for increasing gap widths. ${ }^{14}$ In these conditions, virtually no SWNTs are found bridging gaps wider than $3 \mu \mathrm{m}$. This directly relates to Figure 1: the wider the gap, the less likely for the SWNT to find an anchoring point. The effect can be exploited to control the SWNT position and bridging density, since closer tips increase the likelihood of having SWNTs bridging the gap, whereas sharper tips decrease the likelihood of having multiple SWNTs bridging a particular gap (Figure 2a,b). ${ }^{14}$ Longer distances can be spanned by imposing an electric field ${ }^{10}$ or a 


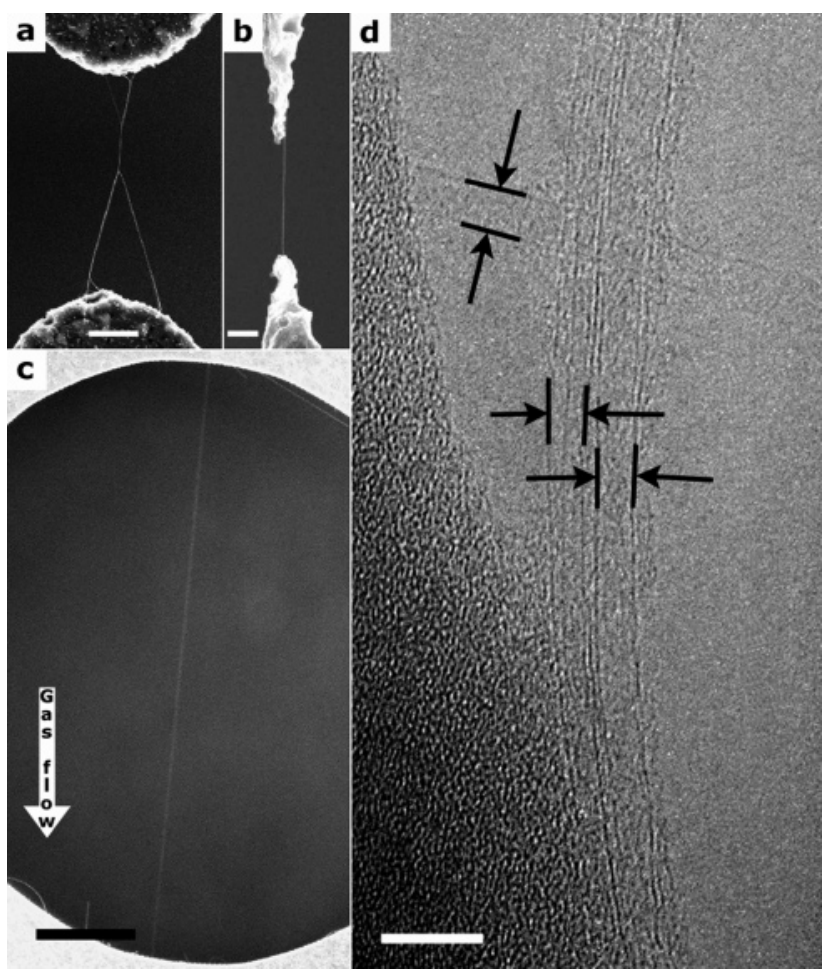

Figure 2. Suspended SWNTs: SEM images of nanotubes grown in static flow conditions at reduced pressure from $\mathrm{Al} / \mathrm{Ni}$ catalyst films (a) bridging rounded tips, scale bar $500 \mathrm{~nm}$, and (b) across sharp tips, scale bar $200 \mathrm{~nm}$. (c) SEM image of gas flow-aligned SWNT grown at atmospheric pressure from Co catalyst bridging a $30 \mu \mathrm{m}$ wide hole, scale bar $5 \mu \mathrm{m}$. (d) TEM image of SWNTs grown at atmospheric pressure from $\mathrm{Fe}$ catalyst crossing a $2 \mu \mathrm{m}$ gap on a SiOx membrane, scale bar $5 \mathrm{~nm}$.

preferential gas flow, ${ }^{13}$ as shown in Figure 2c. Here the growth takes place in an atmospheric pressure furnace under a constant gas flow, which is found to induce the bridging of at least 30 $\mu \mathrm{m}$ gaps.

Another consequence of the observed motion is the bundling of nanotubes. While waving and pivoting (Figure 1), SWNTs are likely to come into contact with each other and form bundles due to vdW forces, as shown in Figure 2a,d. On the other hand, the vdW interaction with the substrate straightens them (Figures $2 b, c)$. This accounts for the tautness commonly observed for suspended nanotubes (see also videos in Supporting Information). ${ }^{13,23}$ Figure $2 \mathrm{~d}$ shows a typical TEM image recorded at the edge of a perforated $\mathrm{SiO}_{x}$ membrane: some nanotubes are found spanning the gap straight, some bundle together forming Y-junctions near the support, and some closely follow the edge of the support before spanning the gap, presumably to decrease the slack of the suspended structure. ${ }^{23}$ The substrate-nanotube interactions can be found to be so strong as to cause SWNTs to buckle. ${ }^{23}$

So far, we have provided evidence for interactions taking place when a nanotube is suspended. More commonly, SWNTs are grown on planar substrates. We now analyze if nanotubes grow in actual close contact with the substrate or rather are partially afloat, as for the suspended case. The influence of etched surface barriers on SWNT alignment is investigated by patterning catalyst films as lines parallel to, but not overlapping the barriers (see Figure 3a,b). SWNT synthesis is carried out in constant gas flow at atmospheric pressure, with free (Figure 3a,d) and obstructed (Figure 3c) gas flows over the substrate surface and in a static atmosphere at reduced pressure (Figure $3 b)$. On the same sample obtained in free gas flow conditions

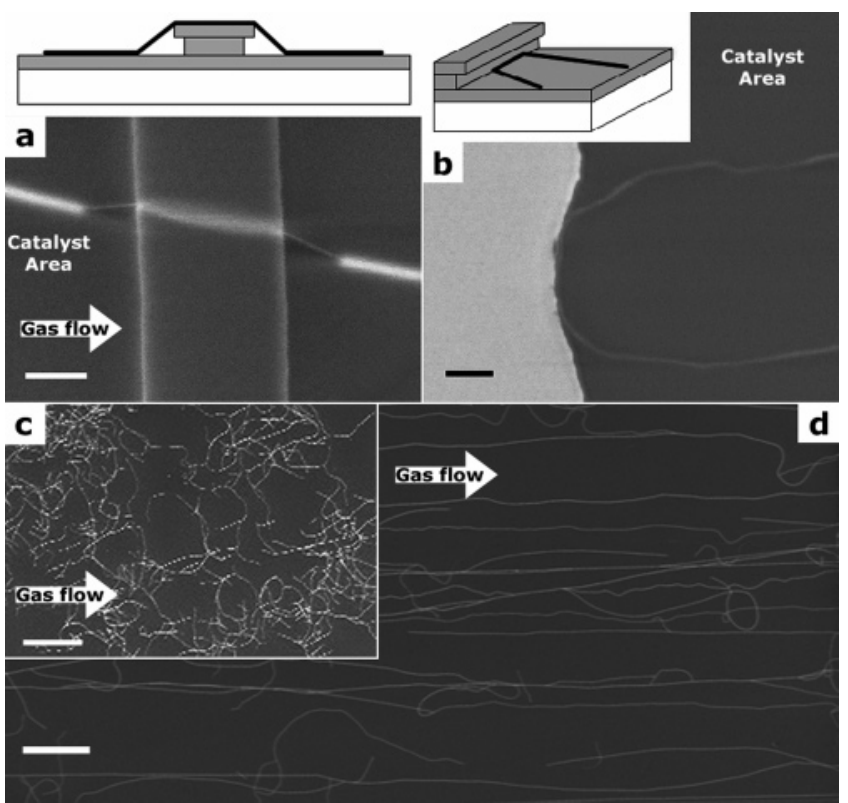

Figure 3. SEM images of SWNTs grown at various CVD conditions. (a) SWNT grown in constant gas flow at atmospheric pressure crossing over an undercut barrier in SOI (flying mode growth), scale bar 200 $\mathrm{nm}$, tilt $30^{\circ}$, (b) SWNT grown in static atmosphere at reduced pressure stopped by an undercut barrier (crawling mode growth), scale bar 500 $\mathrm{nm}$, (c) SWNTs grown with suppressed gas flow (see text) at atmospheric pressure, scale bar $20 \mu \mathrm{m}$, (d) flow-aligned SWNTs grown in constant gas flow at atmospheric pressure, scale bar $40 \mu \mathrm{m}$. Insets in panels $\mathrm{a}$ and $\mathrm{b}$ are schematics illustrating the SEM picture geometries: crystalline silicon in white, silicon dioxide in gray, and SWNT in black.

at atmospheric pressure, we find flow-aligned straight SWNTs seemingly unaffected by the undercut barriers (as in Figure 3a), and SWNTs that do not pass over these barriers and remain entirely within the region where the catalyst was deposited (as in Figure 3b). Free gas flow over the substrate surface promotes the overcoming of barriers and alignment (Figure 3a,d) similarly to what observed in Figure 2c. Flow aligned SWNTs can be extremely long and straight (Figure $3 \mathrm{~d}$ ) with lengths of $\sim 1 \mathrm{~mm}$ routinely achieved for $15 \mathrm{~min}$ syntheses. This alignment is found to be insensitive to the effects of gravity, as one would expect from the negligible mass of the nanotubes. Flow alignment is not found at reduced pressures (Figure 3b) and can be suppressed in a standard atmospheric pressure furnace when the constant gas flow over the substrate surface is obstructed (Figure 3c). In the latter case, to suppress the flow alignment, the sample is placed catalyst-down over a flat crystalline silicon support coated by thermal amorphous silicon dioxide. This reduces the effect of the gas flow while retaining gas diffusion pathways within the microfissures existing between the surfaces in order to feed the growth. Under such conditions, SWNTs are typically not only constrained by the barriers but are shorter and not straight (Figure 3b,c).

Following these observations, we propose a distinction of growth modes not only between tip and base growth but also between crawling and flying (Figure 4). Crawling SWNTs are tip-grown and fully surface-bound, mainly subject to vdW forces and substrate-induced alignment forces (Figure 3b,c). Flying SWNTs are base- or tip-grown and partially surface-bound (Figure 3a,d). The forces acting upon them can be due to vdW interactions, thermal fluctuations, buoyant lift, gas flow, and externally applied electric fields. As shown in Figures 1 and 2 and the Supporting Information videos, flying SWNTs easily anchor themselves to a nearby surface feature. 


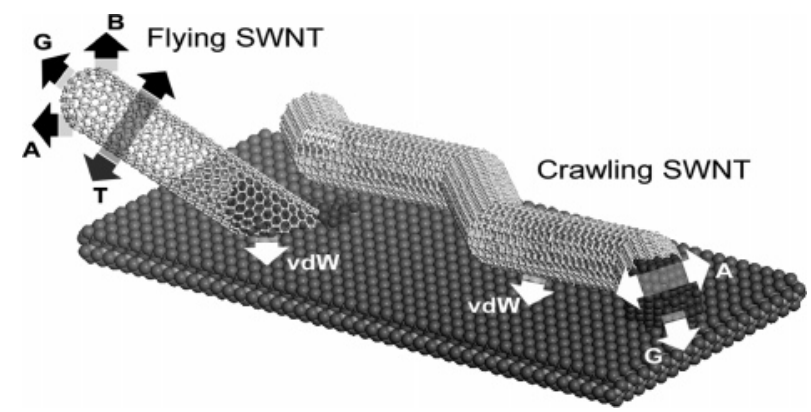

Figure 4. Schematic views of crawling and flying SWNTs with the relevant forces acting upon them: van-der-Waals attraction (vdW), growth extrusion $(G)$, alignment forces $(A)$, thermal fluctuations $(T)$ and buoyant lift $(B)$.

Crawling nanotubes tend to be nonstraight, short, and potentially more defective on amorphous or rough surfaces. This follows from the likelihood of the growing nanotube end to encounter an obstacle that can either change the direction of growth or stop growth entirely. A tip growth mechanism dominates for crawling tubes, since for base growth an increase in SWNT length results in increasingly strong vdW interactions with the substrate. Crawling SWNTs are not able to overcome large/undercut barriers (Figure $3 \mathrm{~b}$ ), as this would require an unlikely combination of sharp kinks in the body of the nanotube. Atomically flat substrates can improve the alignment of crawling nanotubes and have been shown to be effective for the growth of long SWNTs. ${ }^{11}$ Alignment has been shown along atomicheight step edges ${ }^{11,16}$ or along the substrate's crystal orientation. ${ }^{24-26} \mathrm{Yu}$ et al. postulate a raised-head mechanism similar to the crawling growth mode, but with a slightly raised SWNT tip, held separated from the surface due to electrostatic repulsion. ${ }^{26}$ Though we do not exclude this possibility, we still categorize this growth as crawling mode, given the close intimacy between SWNT and substrate, which prevents gas flow alignment.

Flying nanotubes are generally found to be longer and, whenever the appropriate conditions are present, straight and aligned. Being afloat limits substrate interactions and hence decreases the likelihood of pinning to an obstacle and of premature catalyst poisoning. This is supported by experiments of Huang et al., where nanotubes in slow heating conditions are short and not flow aligned (crawlers), but whenever a vertical electric field is applied to keep SWNTs afloat, the SWNTs are longer and flow aligned. ${ }^{15} \mathrm{~A}$ flying SWNT growth mode is compatible with both tip-growth and base-growth. The straightness of flying SWNTs varies with alignment method and experimental conditions. Catalyst patterning over raised structures $^{21,27}$ or a vertical electric field ${ }^{15}$ were shown to increase the alignment yield. Gas flow aligned nanotubes are generally found to be straight over length scales varying from several tens of micrometers (Figure $3 \mathrm{~d}$ ) to centimeters ${ }^{28}$ but may include loops or be U-shaped. ${ }^{8,13}$ The degree of straightness reflects the dynamic conditions in the gaseous CVD environment: the more laminar the gas flow, the better the alignment. ${ }^{9,28}$ A highly laminar flow was reported to result in centimeter-long, straight SWNTs. ${ }^{9,28}$ This length scale is believed to originate from buoyant forces preventing the growing nanotube from completely adhering to the substrate. ${ }^{15,28}$ The presence of loops can be induced by the rapid attachment of the flying SWNTs to the substrate, whereas U-shaped nanotubes can originate from the pinning of the SWNT's tip while growth at its base continues (see also video 2 in the Supporting Information). ${ }^{13}$ Suppressing laminar flow results in loss of alignment: by imposing static
CVD conditions, with turbulent gas flow or by arranging the substrate catalyst-side-down on a support in such a way as to hinder laminar gas flow over the catalyst but still retain diffusive pathways allowing for growth (Figure 3c). Hindering gas flow alignment may be of use in applications where randomly aligned SWNTs are desired or when other alignment schemes such as substrate-mediated alignment are employed.

Besides differences in morphology, flying and crawling nanotubes may be distinguished during SEM analysis of our undercut samples, as they appear to charge differently and have different contrast, even though both types of nanotubes are viewed near one another, on the same type of substrate, and in identical SEM scanning conditions (Figure 3a,b). Flying SWNTs generally appear brighter than crawling SWNTs when imaging at $1 \mathrm{keV}$ with secondary electron detection. SEM contrast can be due to differences in work function or charge accumulation between the SWNT and the substrate. ${ }^{29,30}$ Here, however, both flyers and crawlers are not in contact with any electrodes, and the contrast is unchanged in the regions where the SWNTs are near or on the catalyst. The contrast observed can be interpreted as different charge accumulation in the SWNTs adhering more or less intimately with the underlying substrate. Flying nanotubes may adhere less intimately since they are straight and taut throughout their length.

\section{Conclusions}

Two growth modes were distinguished based on the intimacy of the SWNT-substrate contact and related to the catalyst particle dynamics during catalytic CVD. A tip growth mechanism dominates for crawling nanotubes, which follow the substrate topography and are stopped at large obstacles. Flying nanotubes are generally longer and straighter, can be aligned with the gas flow and external fields, span gaps, and fly over obstacles. The catalyst particle can be either in the tip or base of the flying SWNT. For base growth, the dynamic catalyst crystallite reshaping contributes to nanotube pivoting. Catalyst particle size and support/feed gas interactions have been shown to influence the catalyst dynamics. ${ }^{3-7}$ Here we show that CVD conditions and substrate topography can be additionally optimized to keep anchored SWNTs afloat and hence facilitate their alignment and extend catalyst lifetime.

Acknowledgment. This work was supported by the EU project CANAPE. A.J. and C.S. acknowledge funding from the Swiss National Science Foundation (Grant No. 200021-108059/ 1) and the ETH Zurich (Grant No. TH-18/03-1). F.C.-S. from CONACyT Mexico. A.C.F. acknowledges funding from The Leverhulme Trust. S.H. acknowledges funding from Peterhouse, Cambridge. A.C.F., C.D., and S.H. acknowledge funding from The Royal Society.

Note Added after ASAP Publication. Due to a production error, this manuscript was originally published on October 17, 2007 with incorrect corresponding author contact information. The contact information was corrected, and the manuscript was reposted on October 23, 2007.

Supporting Information Available: ETEM videos (Quicktime, H.263) of Ni catalyzed SWNTs grown at $600{ }^{\circ} \mathrm{C}$ in $\mathrm{C}_{2} \mathrm{H}_{2}$ at $8 \times 10^{-3}$ mbar. Video 1 shows a SWNT pivoting and bridging a nearby support feature. Video 2 shows a SWNT growing after attachment. This material is available free of charge via the Internet at http://pubs.acs.org. 


\section{References and Notes}

(1) Kong, J.; Soh, H. T.; Cassell, A. M.; Quate, C. F.; Dai, H. Nature 1998, 395, 878 .

(2) Helveg, S.; Lopez-Cartes, C.; Sehested, J.; Hansen, P. L.; Clausen, B. S.; Rostrup-Nielsen, J. R.; Abild-Pedersen, F.; Norskov, J. K. Nature 2004, 427, 426.

(3) Hansen, P. L.; Wagner, J. B.; Helveg, S.; Rostrup-Nielsen, J. R.; Clausen, B. S.; Topsoe, H. Science 2002, 295, 2053.

(4) Cantoro, M.; Hofmann, S.; Pisana, S.; Scardaci, V.; Parvez, A.; Ducati, C.; Ferrari, A. C.; Blackburn, A. M.; Wang, K. -Y.; Robertson, J. Nano Lett. 2006, 6, 1107.

(5) Pisana, S.; Cantoro, M.; Parvez, A.; Hofmann, S.; Ferrari, A. C.; Robertson, J. Physica E 2007, 37, 1.

(6) Wang, Y.; Luo, Z.; Li, B.; Ho, P. S.; Yao, Z.; Shi, L.; Bryan, E. N.; Nemanich, R. J. J. Appl. Phys. 2007, 101, 124310.

(7) Hofmann, S.; Sharma, R.; Ducati, C.; Du, G.; Mattevi, C.; Cepek, C.; Cantoro, M.; Pisana, S.; Parvez, A.; Cervantes-Sodi, F.; Ferrari, A. C.; Dunin-Borkowski, R.; Lizzit, S.; Petaccia, L.; Goldoni, A.; Robertson, J. Nano Lett. 2007, 7, 602.

(8) Huang, S.; Cai, X.; Liu, J. J. Am. Chem. Soc. 2003, 125, 5636.

(9) Hong, B. H.; Lee, J. Y.; Beetz, T.; Zhu, Y.; Kim, P.; Kim, K. S. J. Am. Chem. Soc. 2005, 127, 15336.

(10) Joselevich, E.; Lieber, C. M. Nano Lett. 2002, $2,1137$.

(11) Kocabas, C.; Hur, S.-H.; Gaur, A.; Meitl, M. A.; Shim, M.; Rogers, J. A. Small 2005, 1, 1110 .

(12) Lefebvre, J.; Homma, Y.; Finnie, P. Phys. Rev. Lett. 2003, 90, 217401.

(13) Huang, L.; White, B.; Sfeir, M. Y.; Huang, M.; Huang, H. X.; Wind, S.; Hone, J.; O’Brien, S. J. Phys. Chem. B 2006, 110, 11103.
(14) Jungen, A.; Hofmann, S.; Meyer, J. C.; Stampfer, C.; Roth, S.; Robertson, J.; Hierold, C. J. Micromech. Microeng. 2007, 17, 603.

(15) Huang, S.; Woodson, M.; Smalley, R.; Liu, J. Nano Lett. 2004, 4, 1025 .

(16) Ismach, A.; Kantorovich, D.; Joselevich, E. J. Am. Chem. Soc. 2005, 127,11554

(17) Murakami, Y.; Chiashi, S.; Miyauchi, Y.; Hu, M.; Ogura, M.; Okubo, T.; Maruyama, S. Chem. Phys. Lett. 2004, 385, 298.

(18) Sharma, R. J. Mater. Res. 2005, 20, 1695.

(19) Sharma, R.; Rez, P.; Treacy, M. M. J.; Stuart, S. J. J. Electron Microsc. 2005, 54, 231.

(20) Sharma, R.; Iqbal, Z. Appl. Phys. Lett. 2004, 84, 990

(21) Li, S.; Yu, Z.; Rutherglen, C.; Burke, P. J. Nano Lett. 2004, 4, 2003.

(22) Krishnan, A.; Dujardin, E.; Ebbesen, T. W.; Yianilos, P. N.; Treacy, M. M. J. Phys. Rev. B 1998, 58, 14013.

(23) Abrams, Z. R.; Hanein, Y. J. Phys. Chem. B 2006, 110, 21419.

(24) Ago, H.; Nakamura, K.; Ikeda, K.; Uehara, N.; Ishigami, N.; Tsuji, M. Chem. Phys. Lett. 2005, 408, 433.

(25) Han, S.; Liu, X.; Zhou, C. J. Am. Chem. Soc. 2005, 127, 5294

(26) Yu, Q.; Qin, G.; Li, H.; Xia, Z.; Nian, Y.; Pei, S.-S. J. Phys. Chem. $B$ 2006, 110, 22676.

(27) Hongo, H.; Nihey, F.; Ochiai, Y. J. Appl. Phys. 2007, 101, 024325.

(28) Jin, Z.; Chu, H.; Wang, J.; Hong, J.; Tan, W.; Li, Y. Nano Lett. 2007, 7, 2073.

(29) Zhang, R. Y.; Wei, Y.; Nagahara, L. A.; Amlani, I.; Tsui, R. K. Nanotechnology 2006, 17, 272.

(30) Jesse, S.; Guillorn, M. A.; Ivanov, I. N.; Puretzky, A. A.; Howe, J. Y.; Britt, P. F.; Geohegan, D. B. Appl. Phys. Lett. 2006, 89, 013114. 